\title{
Fiber-based measurement of temporal intensity and phase profiles of an optical telecommunication pulse through self-phase modulation
}

\author{
Killian Baudin, Frédéric Audo and Christophe Finot * \\ Laboratoire Interdisciplinaire CARNOT de Bourgogne, \\ UMR 6303 CNRS-Université de Bourgogne-Franche-Comté, \\ 9 Av. A. Savary, BP 47 870, 21078 DIJON Cedex, FRANCE \\ *christophe.finot@u-bourgogne.fr
}

\begin{abstract}
We propose and experimentally validate an all-fiber based approach to characterize the phase and intensity profiles of optical pulses. Based on three optical spectra affected by different levels of self-phase modulation, we were able to reconstruct the temporal details of pulses typical of optical telecommunications.
\end{abstract}

Keywords: nonlinear optics, fiber optics, pulse characterization 


\section{Introduction}

Characterization of amplitude and phase of optical pulses remains a critical and challenging issue for numerous applications ranging coherent control to optical telecommunications [1, 2]. To provide accurate information, a wide set of approaches has been developed based on different optical signals recorded in the temporal or/and spectral domains. As just a few examples of such all-optical technics, we can mention the algorithms following Gerbech-Saxton initial idea [3] based on the intensity profiles directly recorded in the temporal and spectral domains, the PICASSO method [4] based on a spectrum and autocorrelation measurements, the use of transport intensity equation [5], the Frequency Resolved Optical Gating approach [6] or the spectral phase interferometry technics [7]. The level of complexity of the optical setup and the associated algorithms may significantly vary, as well as the complexity of the pulses that can be conveniently characterized.

More recently, a new and simple method that takes advantage of spectral measurements of pulses affected by a known level of self-phase modulation (SPM) has been introduced [8] to characterize the temporal intensity and phase profiles: using ultrahigh peak-power femtosecond pulses experiencing Kerr nonlinearity upon propagation in two mm-thick polyethylene terephthalate films, the authors were able to reconstruct efficiently the temporal properties on the input waveform. We investigate in this contribution the extension of this principle to an architecture based on highly nonlinear optical fibers. After having recalled the basis of this method and described the experimental setup we have implemented, we demonstrate that pulses with 
durations around 100 ps and peak power as low as a few Watts can be efficiently characterized both in intensity and phase.

\section{Principle of operation and experimental setup}

Self-phase modulation (SPM) affects any pulse propagating in a Kerr media such as a centrosymmetric nonlinear crystal, an amorphous polymer thermoplastics or an optical fiber: the phase profile of the pulse acquires an additional phase that is proportional to the temporal intensity profile of the input pulse. As a direct consequence, the optical spectrum of the pulse is modified and can experience either spectral broadening or narrowing depending on the temporal chirp of the input pulse $[9,10]$. As the spectrum affected by SPM depends both on the temporal intensity and phase input profiles, it sounds relevant to try to reconstruct the input pulses from an initial output spectrum and a spectrum affected by a known level of SPM usually characterized by the Bintegral. This forms the basis of an ultrashort characterization method initially proposed in the 90s where femtosecond $\mathrm{mJ}$ pulses where efficiently characterized using a cm-thick fused silica plate [11, 12]. However, this approach may require an assumption on the value of the input peak-power and therefore on the input pulse shape. This guess value may affect the result obtained after reconstruction. In order to overcome this drawback, a more robust algorithm has been proposed taking advantage of a set of three measured spectra: an initial spectrum, a second spectrum affected by a nonlinearity $B$ and a third spectrum affected by a nonlinearity that is twice the 
previous one [8]. Numerical and experimental results have validated this approach using $50 \mathrm{fs}$ pulses propagating in $\mathrm{mm}$-thick nonlinear films.

Our idea is to extend and adapt this recent work to pulses with much longer durations and much lower peak powers (several tens of picoseconds, a few Watts) typical of optical telecommunications. To this aim, the all-fiber experimental setup we have implemented is sketched in Fig. 1 and relies on commercially available devices exclusively. The waveform under test is generated by a continuous wave laser emitting in the conventional band of the telecommunications $(1550 \mathrm{~nm})$ that is then intensity modulated by a LithiumNiobate modulator (IM) driven by a pattern generator delivering electrical pulses with durations around $100 \mathrm{ps}$ at a repetition rate around $100 \mathrm{MHz}$. In order to test the impact of a dispersive element, we also include for some measurements a spool of dispersion compensating fiber (DCF) with a length of $13 \mathrm{~km}$ and a value of dispersion of $-120 \mathrm{ps} / \mathrm{km} / \mathrm{nm}$. The characterization stage includes an erbium-doped fiber amplifier (EDFA) in order to reach the required input peak power. A sufficient SPM is accumulated thanks to the long interaction length experienced in a highly nonlinear optical fiber (HNLF) with a Kerr coefficient $\gamma=10 \mathrm{~W} / \mathrm{km}$, a length $L=500 \mathrm{~m}$ and a reduced dispersion of $\neg 1 \mathrm{ps} / \mathrm{km} / \mathrm{nm}$ that can be neglected for the pulse durations under study. In the context of optical fiber, the B-integral can be as a first approximation evaluated as $B=\gamma P_{0} L$ with $P_{0}$ the input pulse peak power. Contrary to [8] where two nonlinear elements were involved and the input beam was divided, we made the choice of a single Kerr element. Consequently, the spectra corresponding to two values of $B$ where recorded by varying the power launched into the fiber using a variable optical attenuator (VOA). 
The input temporal and spectral intensity profiles were characterized using a $70 \mathrm{GHz}$ photodiode associated with a $70 \mathrm{GHz}$ sampling electrical oscilloscope and a high-resolution optical spectrum analyzer. The use of a complex optical spectrum analyzer (APEX AP2443B) also enables us to have access to an independent measurement of the temporal intensity and phase. At the output of the HNLF, two spectra affected by SPM are also recorded with a high-resolution spectrum analyzer. Let us remark that contrary to [8], our experimental devices are not adapted to single shot measurements.

\section{Experimental results}

We have experimentally tested various waveforms. Results are summarized in Fig. 2, 3 and 4 for a selection of three different profiles. For each input shape, three optical spectra are recorded: the spectra without SPM, the spectra affected by SPM with a B-integral of 2 rad (panels (b)) and finally the spectra affected by SPM of 4 rad (panels (c)). These levels of SPM correspond to peak powers launched in the HNLF of a few Watts only. In order to make the numerical treatment easier, the coherent component of the spectra that is linked to the finite extinction ratio of the optical intensity modulator is numerically removed and the comb-like spectrum of the periodic signals recorded on the high-resolution spectrum is used to correct the measurements from the amplified spontaneous emission. The numerical iterative algorithm that is used to retrieve the temporal properties of the pulse is strictly identical to the one detailed in the initiating work of [8] and inspired by Gerchberg-Saxton-like approaches. 
The first waveform we present (Fig. 2) is a Gaussian-like pulse obtained using a $5.4 \mathrm{GHz}$ electrical filter on the signal driving the intensity modulator. The resulting pulse has 117 ps duration. Fig. 2(a) shows that the retrieved temporal intensity profile is in remarkable agreement with the profile directly measured on the high bandwidth photodiode as well as the profile provided by the APEX AP2443B. The spectral profiles reconstructed from the algorithm are also in perfect agreement with the ones that are measured directly. The phase of the pulse under study is rather flat, indicating the waveform is quite close to the Fourier transform limit. The processing of the phase is validated by the comparison with the profile provided by the APEX device, the agreement between the two phase profiles being good.

In order to test further our setup, the second waveform we study (Fig. 3) is a flat-top pulse with sharper edges. Once again, the retrieved temporal intensity profile is in remarkable agreement with the super-Gaussian like profile recorded directly on the photodiode. This confirms the ability of the algorithm to process accurately pulses with sharp fronts and to efficiently reconstruct a waveform that is consistent with the three recorded spectra.

We finally tested in Fig. 4 a Gaussian-like pulse affected by second order dispersion imprinted through the propagation in an $13 \mathrm{~km}$ long DCF. Once again, the temporal intensity profiles retrieved from the algorithm clearly match the results separately recorded on the photodiode and the results provided by the APEX device: all the measurements reproduce the same slight temporal asymmetry in the wings. The phase profile is close from the results measured with the complex optical spectrum analyzer and is in agreement with the parabolic phase that can be inferred from the propagation distance in the DCF 
fiber. These results were obtained in the case of a phase induced by a normally dispersive fiber. We have numerically checked that the iterative algorithm was efficient for characterizing a pulse affected by an anomalous dispersive element that may lead to a more complex spectral dynamics in the HNLF with a stage of nonlinear spectral compression [10].

\section{Conclusions}

We have extended the approach initially proposed by E. A. Anashkina et al [5] to measure the intensity and phase profiles of pulses typical of optical telecommunications. We have implemented an alignment-free setup involving a highly nonlinear optical fiber instead of the nonlinear films initially used in [8]. By using peak powers of only a few Watts, we have also shown that the approach was not restricted to TW or PW class lasers. Our demonstration has been achieved with pulses having a duration around one hundred of picoseconds and we believe that similar performance can be achieved with durations down to a few tens of picoseconds. For pulse durations below ten picoseconds, the chromatic dispersion of the fiber may impair the accuracy of the approach leading to a temporal modification of the intensity profile such as soliton-like compression [13] or wave-breaking induced broadening [14], but the algorithm could be corrected accordingly [12]. Our scheme relies on nonlinear fibers but the footprint of the device could be much more reduced with the potential use of highly nonlinear waveguides such as silicon or chalcogenide $\mathrm{cm}$-long devices $[15,16]$ as long as higher orders of nonlinearity can be ignored. 


\section{Acknowledgements:}

We acknowledge the financial support of the Conseil Regional de Bourgogne (Pari Photcom) and the Program FEDER-FSE Bourgogne 2014-2020. We also thank the funding of the Labex ACTION program (ANR-11-LABX-01-01) and the support of the Institut Universitaire de France. The article has benefited from the PICASSO experimental platform of the University of Burgundy. 


\section{Figure captions:}

Fig. 1 Experimental setup. CW: Continuous Wave; IM: Intensity Modulator; DCF: Dispersion Compensating Fiber; EDFA: Erbium Doped Fiber Amplifier; OVA: Optical Variable Attenuator; HNLF: Highly Nonlinear Fiber; OSA: Optical Spectrum Analyzer; ESO: Electrical Sampling Oscilloscope; COSA: Complex Optical Spectrum Analyzer (APEX AP2443B)

Fig. 2 Experimental results obtained for a Fourier-transform limited Gaussianlike pulse. (a) Temporal intensity and phase profiles obtained after reconstruction based on our approach (solid curve) are compared with the measurements made with an APEX AP2443B device (circles) and the direct measurements made with a photodiode (stars). (b) Measured optical spectra (circles) obtained for B-integral values of 2 and 4 (circles, panel 1 and 2 respectively) are compared with the spectra obtained after reconstruction (solid lines)

Fig. 3 Experimental results obtained for a flat-top pulse. (a) Temporal intensity obtained after reconstruction based on our approach (solid curve) is compared with the direct measurements made with a photodiode (stars). (b) Measured optical spectra (circles) obtained for B-integral values of 2 and 4 (circles, panel 1 and 2 respectively) are compared with the spectra obtained after reconstruction (solid lines)

Fig. 4 Experimental results obtained for a Gaussian-like pulse after propagation in a $13 \mathrm{~km}$ DCF. (a) Temporal intensity and phase profiles obtained after reconstruction based on our approach (solid curve) are compared with the measurements made with an APEX AP2443B device (red circles) and the results that can be expected from the dispersion induced by the DCF (dashed line). (b) Measured optical spectra (circles) obtained for B-integral values of 2 and 4 (circles, panel 1 and 2 respectively) are compared with the spectra obtained after reconstruction (solid lines) 
Figure 1

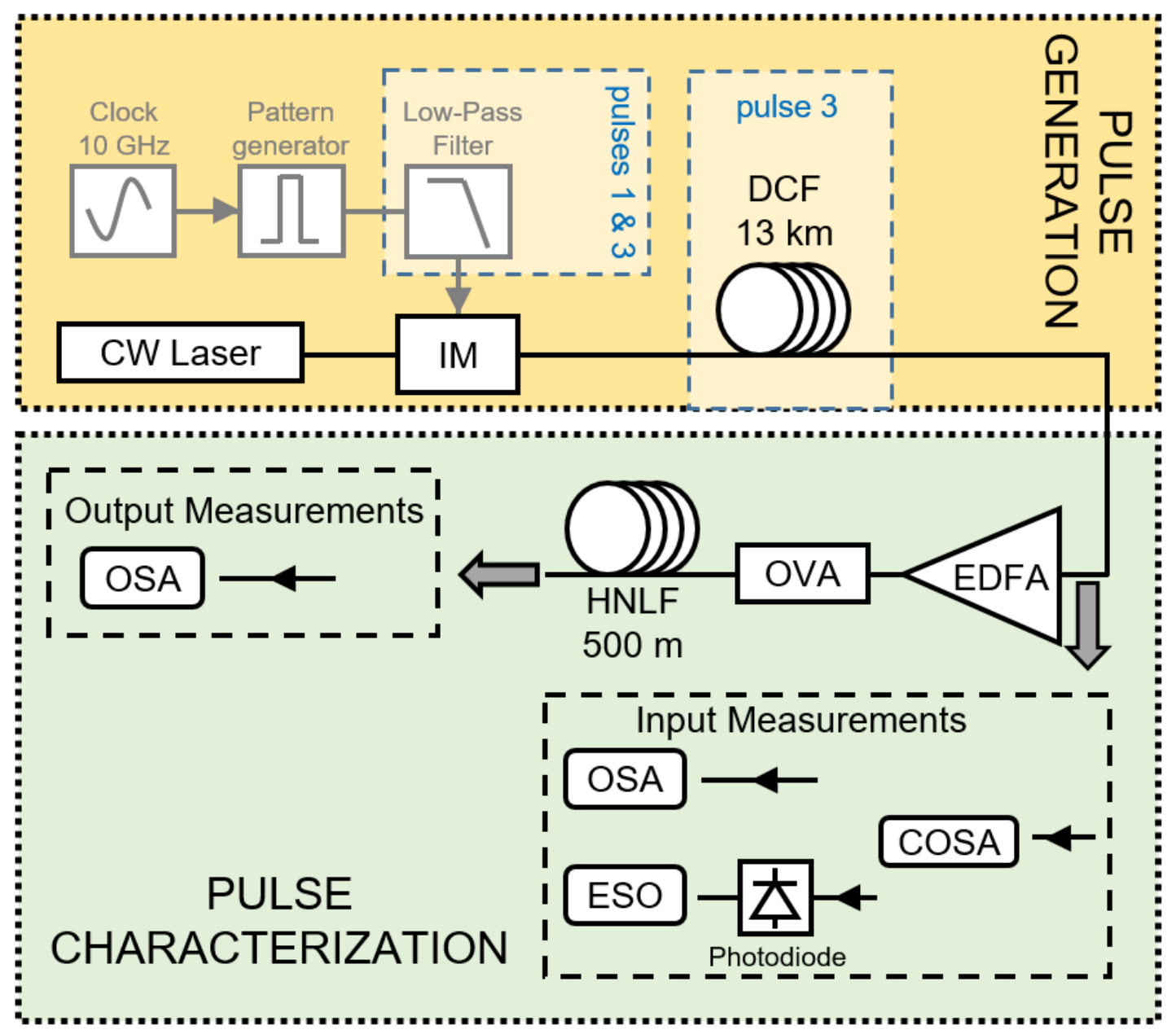


Figure 2
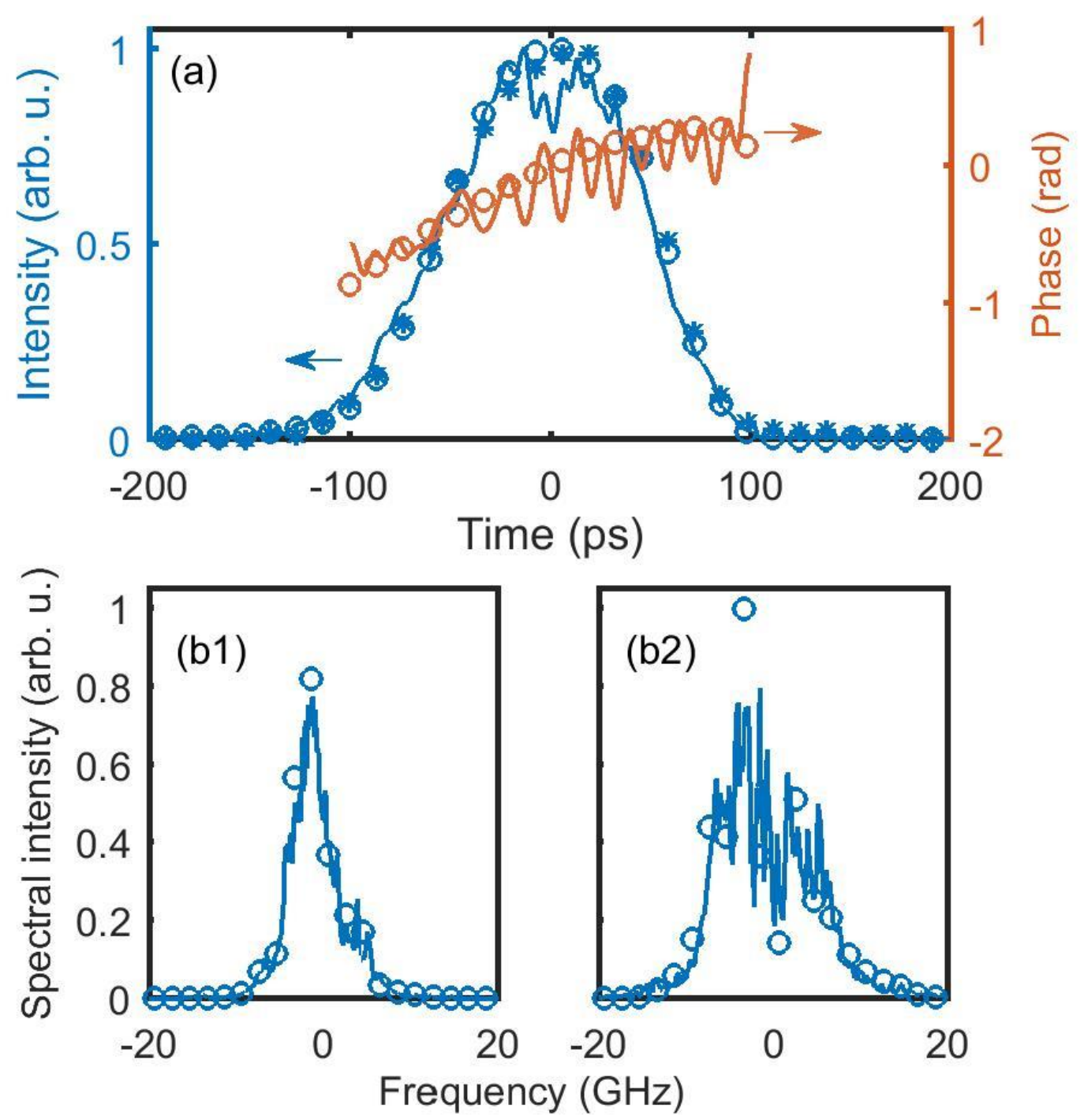
Figure 3
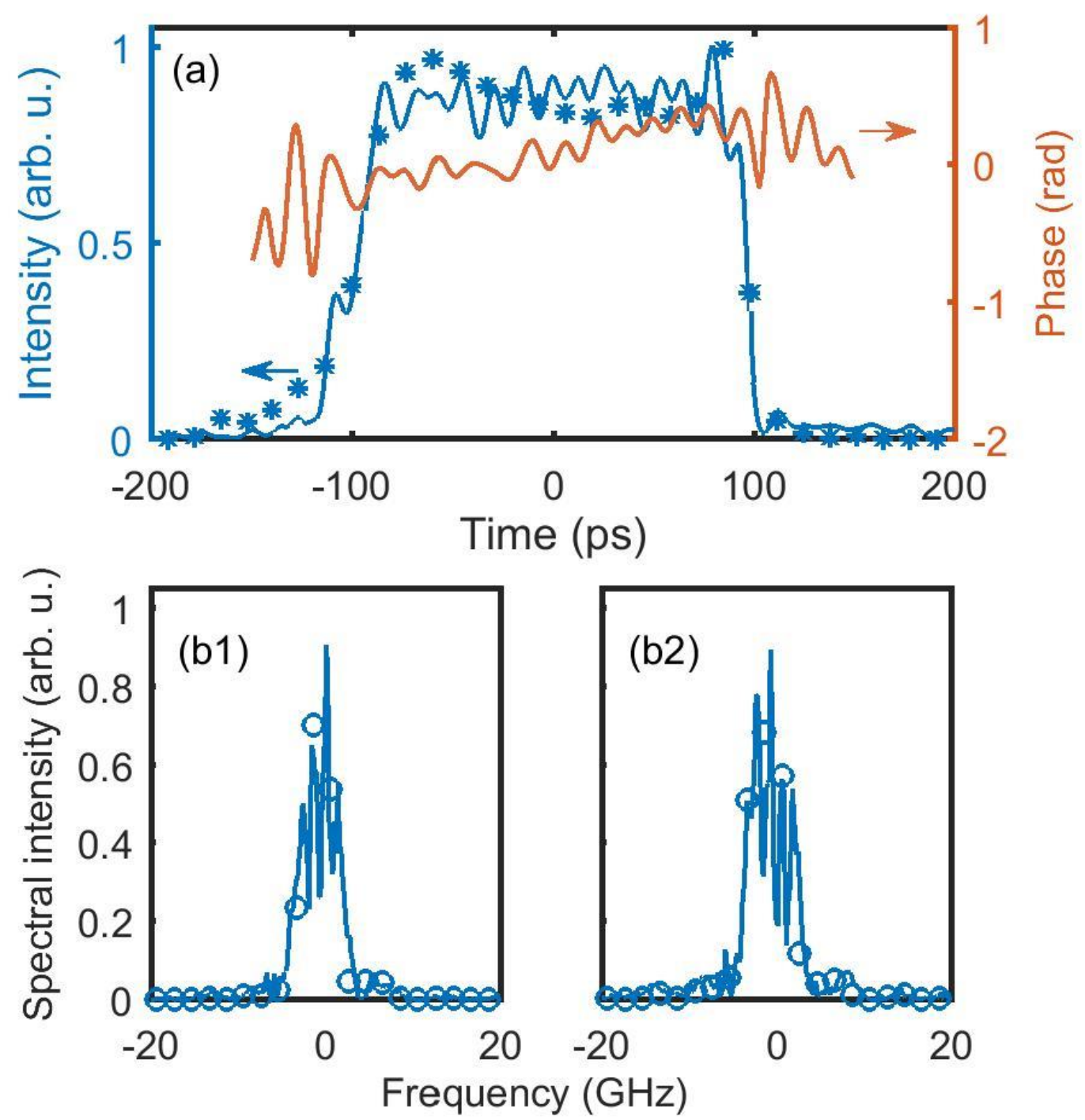
Figure 4
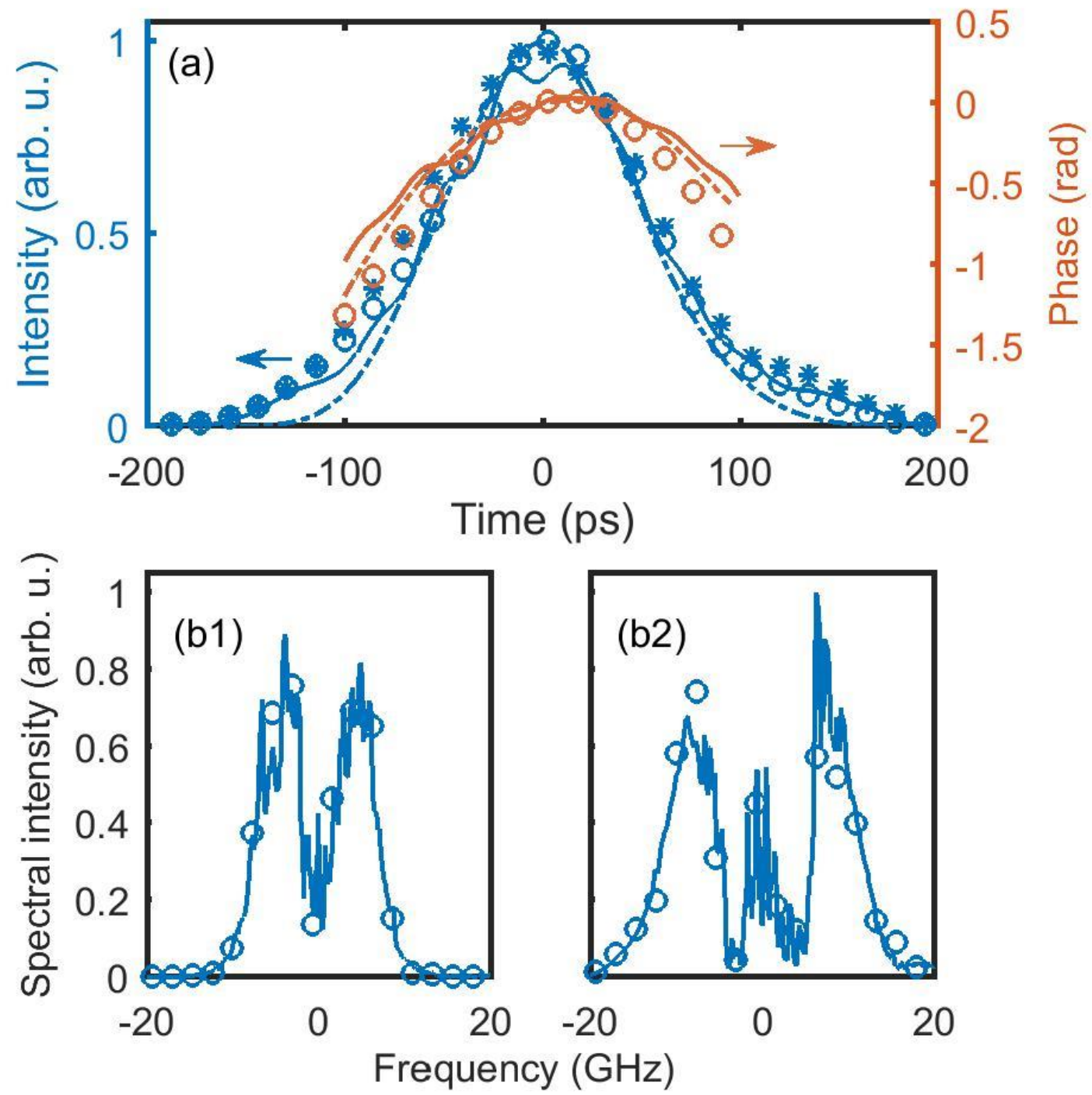


\section{References}

[1] I.A. Walmsley, C. Dorrer, Characterization of ultrashort electromagnetic pulses, Adv. Opt. Photon., 1 (2009) 308-437.

[2] C. Dorrer, High-speed measurements for optical telecommunication systems, IEEE J. Sel. Top. Quantum Electron., 12 (2006) 843-858.

[3] R.W. Gerchberg, W.O. Saxton, A practical algorithm for the determination of the phase from image and diffraction plane pictures, Optik 35 (1972) 237-246.

[4] J.W. Nicholson, W. Rudolph, Noise sensivity and accuracy of femtosecond pulse retrieval by phase and intensity from correlation and spectrum only (PICASO), J. Opt. Soc. Amer. B, 19 (2002) 330-339.

[5] C. Dorrer, Characterization of nonlinear phase shifts by use of the temporal transport-of-intensity equation, Opt. Lett, 30 (2005) 3237-3239.

[6] R. Trebino, Frequency-Resolved Optical Gating : the measurement of ultrashort laser pulses, Norwell, MA : Kluwer Academic Publishers, 2000.

[7] C. Iaconis, I.A. Walmsley, Spectral phase interferometry for direct electricfield reconstruction of ultrashort optical pulses, Opt. Lett., 23 (1998) 792-794.

[8] E.A. Anashkina, V.N. Ginzburg, A.A. Kochetkov, I.V. Yakovlev, A.V. Kim, E.A. Khazanov, Single-shot laser pulse reconstruction based on self-phase modulated spectra measurements, Scientific Reports, 6 (2016) 33749.

[9] R.H. Stolen, C. Lin, Self-phase modulation in silica optical fibers, Phys. Rev. A, 17 (1978) 1448-1453.

[10] C. Finot, S. Boscolo, Design rules for nonlinear spectral compression in optical fibers, J. Opt. Soc. Am. B, 33 (2016) 760-767.

[11] B.S. Prade, J.M. Schins, E.T.J. Nibbering, M.A. Franco, A. Mysyrowicz, A simple method for the determination of the intensity and phase of ultrashort optical pulses, Opt. Commun., 113 (1994) 79-84.

[12] E.T.J. Nibbering, M.A. Franco, B.S. Prade, G. Grillon, J.P. Chambaret, A. Mysyrowicz, Spectral determination of the amplitude and the phase of intense ultrashort optical pulses, J. Opt. Soc. Am. B, 13 (1996) 317-329.

[13] L.F. Mollenauer, R.H. Stolen, J.P. Gordon, Experimental observation of picosecond pulse narrowing and solitons in optical fibers, Phys. Rev. Lett., 45 (1980) 1095-1098. 
[14] C. Finot, B. Kibler, L. Provost, S. Wabnitz, Beneficial impact of wavebreaking on coherent continuum formation in normally dispersive nonlinear fibers, J. Opt. Soc. Am. B, 25 (2008) 1938-1948.

[15] B.J. Eggleton, B. Luther-Davies, K. Richardson, Chalcogenide photonics, Nat Photon, (2011).

[16] J. Leuthold, C. Koos, W. Freude, Nonlinear silicon photonics, Nature Photonics, 4 (2010) 535-544. 